\title{
Nanometer accuracy statistical interferometric technique in monitoring the short-term effects of exogenous plant hormones, auxin and gibberellic acid, on rice plants
}

\author{
Mahjabin Kabir ${ }^{1,2}$, Naruke Haruki ${ }^{1}$, UmaMaheswari Rajagopalan ${ }^{3}$, \\ Mikihisa Umehara ${ }^{4}$, Hirofumi Kadono, ${ }^{1, *}$ \\ ${ }^{1}$ Graduate School of Science and Engineering, Saitama University, 255 Shimo Okubo, Sakura-ku, Saitama 338-8570, Japan; \\ ${ }^{2}$ Department of Farm Power and Machinery, Faculty of Agricultural Engineering and Technology, Bangladesh Agricultural \\ University, Mymensingh 2202, Bangladesh; ${ }^{3}$ Shibaura Institute of Technology, 3-7-5 Toyosu, Koto-ku, Tokyo 135-8548, \\ Japan; ${ }^{4}$ Department of Applied Biosciences, Faculty of Life Sciences, Toyo University, 1-1-1 Izumino, Itakura-machi, Ora-gun, \\ Gumma 374-0193, Japan \\ *E-mail: kadono@mail.saitama-u.ac.jp Tel \& Fax: +81-48-858-3873
}

Received January 10, 2020; accepted February 25, 2020 (Edited by K. Suzuki)

\begin{abstract}
Statistical interferometric technique (SIT) is a highly sensitive, high speed non-contact, and non-destructive optical technique developed by our group capable of measuring instantaeoues sub-nanometer displacements. SIT applied to plant leaf elongation revealed nanometric intrinsic fluctuaitons (NIF) that are robust and sensitive to variations in the environment making NIF as a measure of healthiness of the plants. In this study, exogenous plant hormones, auxin (2,4-dichlorophenoxyacetic acid-2,4-D), and gibberellic acid $\left(\mathrm{GA}_{3}\right)$, along with an auxin transport inhibitor 2,3,5-triiodobenzoic acid-TIBA, that affect plant growth were used to investigate their effects on NIF. Rice (Oriza sativa)

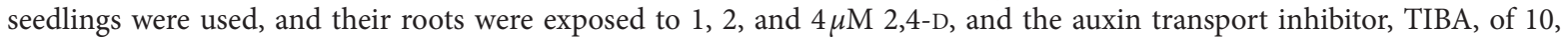
and $20 \mu \mathrm{M}$ for $22 \mathrm{~h}$ and $\mathrm{GA}_{3}$ solution of different concentrations of 10,40 , and $100 \mu \mathrm{M}$ for $5 \mathrm{~h}$. Results showed significant increment in NIF for $1 \mu \mathrm{M}$ and reduction for $4 \mu \mathrm{M}$ 2,4-D while applicaiton of both 10 , and $20 \mu \mathrm{M}$ TIBA led to reduction in NIF. On the other hand, significant increment in NIF for $40 \mu \mathrm{M}$, and a significant reduction at a higher concentration of $100 \mu \mathrm{M}$ for 5 hours of $\mathrm{GA}_{3}$ were also observed in comparison to those of control. Our results indicate that NIF as revealed by SIT could show both the positive and negative effects depending on the concentration of exogenous hormones, and transport inhibitors. Results suggest that SIT could be a valuable tool being sensitive enough to speedily assess the effects of plant growth hormones.
\end{abstract}

Key words: auxin, gibberellic acid, nanometric intrinsic fluctuation, statistical interferometric technique, TIBA.

\section{Introduction}

In general, optical techniques are non-invasive and noncontact, and so they are widely applied in visualizing biological things. At the same time, an optical interferometer is an optical technique that can be applied to study the surface shape or distance measurements at a high accuracy. An optical interferometer is based on the interference of reflected light coming from a reference mirror and an object that is optically flat, i.e., the surface roughness is much less than the wavelength of light (Hariharan 1985). However, application of such an interferometer has a limitation when considering its implementation to the study of the biological tissues due to the strong scattering properties such as a plant leaf surface. This kind of unwanted scattered light forms a random pattern commonly known as laser speckles (Dianty 1975). There exists a few attempts of using such optical interferometers in plant growth measurement at higher resolutions (Briers 1977; Fox and Puffer 2008; Jiang and Stadue 1989); however, remained impractical due to the appearance of speckle noise.

On the other hand, in our group, we have been developing an interferometer called, statistical interferometric technique (SIT) which uses a completely random speckle field as a reference instead of a plane reference wave as generated by an optically flat mirror. The technique is based on the fact that a fully developed

Abbreviations: SIT, statistical interferometric technique; NIF, nanometric intrinsic fluctuations; 2,4-D, 2,4-dichlorophenoxyacetic acid; TIBA, 2,3,5-triiodobenzoic acid; $\mathrm{GA}_{3}$, gibberellic acid; RER, relative elongation rate; NSD, normalized standard deviation; ANSD, average normalized standard deviation; PZT, piezoelectric transducer; CCD, charge-coupled device.

This article can be found at http://www.jspcmb.jp/

Published online July 31, 2020 
speckle field could be used as a reference in a statistical sense with the probability density function of random speckle phase following a uniform distribution from $-\pi$ to $+\pi$ (Kadono and Toyooka 1991; Kobayashi and Kadono 2010). This unique principle of the method makes it possible to apply for the biological object such as plant with very high sensitivity and a simple optical system. In addition to common advantages of an optical interferometric technique, i.e., non-contact and nondestructive measurements, SIT is capable of measuring nanometric displacements at a few hundred msec resolution within the plant surface from a few hours to extended periods of time such as a few days (Kadono and Toyooka 1991; Kobayashi and Kadono 2010). In previous studies, SIT was used effectively to measure the minute root elongation of Japanese red pine fine roots infected by ecto-mycorrhiza (Rathnayake et al. 2007, 2008).

SIT has been applied to measure leaf elongation under different environmental conditions. Application of SIT to plant leaf surface elongation measurements at a temporal resolution of $500 \mathrm{~ms}$ and at an accuracy of nanometer revealed that the elongation was observed to have nanometric level fluctuations. These fluctuations were named as nanometric intrinsic fluctuations (NIF) and are found to be sensitive to the environment. In contrast to conventional measurements such as biomass or height, and chemical analysis that take a long period approximately weeks to months and destructive to plant, NIF are found to be sensitive within a few hours.

NIF could be either enhanced or diminished was successfully demonstrated by the presence of harmful substances, Ozone for rice plants (Thilakarathne et al. 2014a) or heavy metal, Cd (De Silva et al. 2016), or favorable amounts of $\mathrm{Zn}$ (De Silva et al. 2017) for Chinese chives. These measurements revealed the potential of SIT in fast monitoring within a few hours about the effects of environmental stress on plant growth.

In this study, we have investigated the role of SIT in speedily assessing the effects of endogenous plant hormones on applying exogenous hormones. It is well known that the effects of endogenous plant hormones could be modulated by the application of exogenous ones (Taiz and Zeiger 2003). For our experiments, we have employed the commonly used two phytohormones, auxin (2,4-dichlorophenoxyacetic acid-2,4-D), and gibberellic acid $\left(\mathrm{GA}_{3}\right)$. Effect of an auxin transport inhibitor 2,3,5-triiodobenzoic acid (TIBA) was used to confirm the inhibitory effect of endogenous auxin in rice plant.

Plant growth hormones are naturally occurring organic substances which actuates plant growth by influencing physiological processes, mainly germination, cell elongation, cell division, flower bud formation, and aging at low concentrations (Davies 2010). Among them, auxin is the first plant hormone that has been confirmed to exist and is known to have many physiological activities (Majda and Robert 2018; Cai et al. 2018; Zhao et al. 2013). As a popular growth stimulator, auxin have been extensively used (Kirpichnikova et al. 2018; Sosnowski et al. 2019; Takahashi et al. 2012; Zhao 2010) to investigate growth influencing parameters. 2,4--D is a synthetic auxin commonly used as herbicide, at lower concentration shows growth promoting behavior, and at higher concentration acts as environmentally unfriendly to plants (Clark and Pazdernik 2016; Pohanish 2015; Tiwari et al. 2019).

The influences of 2,3,5-triiodobenzoic acid (TIBA) on the transport and metabolism of auxin were studied with different plant species (Botía et al. 1992; KarabaghliDegron et al. 1998). Plant system under different concentrations of TIBA was investigated (Begum et al. 2016; Kučerová et al. 2016; Shinkle and Briggs 1984) and found the inhibitory effect of TIBA for auxin signalling.

We have further demonstrated the usage of SIT in monitoring the effects of another plant hormone, gibberellic acid $\left(\mathrm{GA}_{3}\right)$. Gibberellins (GAs) are endogenous plant hormones that are essential for many developmental processes, cellular expansion, flowering, fruit development and so on in plants (Matsukura et al. 1998; Shibaoka 1993; Tanimoto 2005, 2012). Gibberellins are known to affect leaf elongation in many plant species with more than 130 GAs identified in plants, and only a few GAs including $\mathrm{GA}_{3}$ being biologically active (Furukawa et al. 1997; Yamaguchi 2008). GA acts by removing the limitation to growth and promotes cell wall elongation in different plant species depending on concentrations (Keyes et al. 1990; Miceli et al. 2019; Tanimoto and Huber 1997; Taylor and Cosgrove 1989), and simultaneously inhibits root growth (Kawamura et al. 1976; Tanimoto 2012). Plant growth response was stimulated by exogenous $\mathrm{GA}_{3}$, was observed with 10 , and $30 \mu \mathrm{M}$ (Brock and Kaufman 1988; Daie 1987), while inhibitory effect was observed under $100 \mu \mathrm{M}$ (Durnam and Jones 1982). Therefore, there exists considerable evidence for the influence of auxin, auxin transport inhibitor TIBA, and $\mathrm{GA}_{3}$ on plant growth.

According to previous study our system can monitor successfully the in plane displacement of plant leaf (De Silva et al. 2016, 2017; Thilakarathne et al. 2014a) under different stress conditions. Although 2,4-D is a synthetic auxin, and cannot be transported between plant cells, can be used to monitor successfully the in-plane elongation of leaf surface under different concentrations. In this experiment, we have measured nanometric relative elongation rate of rice leaves under four different concentrations of 2,4-D, $0,1,2$, and $4 \mu \mathrm{M}$ based on the findings of previous study (Clark and Pazdernik 2016; Pohanish 2015; Shi et al. 2008).

In order to confirm the effects of 2,4-D application, experiments with an auxin transport inhibitor, 2,3,5-triiodobenzoic acid-TIBA, were conducted under 
10 , and $20 \mu \mathrm{M}$. Here the concentrations were chosen based on existing works (Begum et al. 2016; Takeno and Pharis 1982; Zhang et al. 2014) to observe the inhibition effects using SIT. Apart from auxin, another important growth hormone, $\mathrm{GA}_{3}$, was used for monitoring the leaf elongation by SIT at different concentrations. Although $100 \mu \mathrm{M}$ is a higher concentration of $\mathrm{GA}_{3}$ than usual, this concentration was used to monitor the changes in NIF compared with lower concentrations of $\mathrm{GA}_{3}$. On the basis of literature (Brock and Kaufman 1988; Durnam and Jones 1982; Valery et al. 2014) four different concentrations, $0,10,40$, and $100 \mu \mathrm{M}$ were chosen.

\section{Materials and methods}

\section{Plant growth parameters and procedure}

Rice is one of the fastest growing staple foods of more than half of the world's population. In our study a Japanese rice cultivar, Koshihikari, was selected as a sample as it is one of the most widely grown cultivars in Japan (Kobayashi et al. 2018). Rice seeds were first surface sterilized using a Benomyl 50\% solution (Hokkyo Industry Corp. Ltd., Tokyo, Japan) for $24 \mathrm{~h}$ at room temperature and then rinsed with distilled water. After that, the seeds were placed in a small plastic box with watered tissue paper and kept in a growth chamber (Conviron, Controlled Environmental Ltd., Winnipeg, Manitoba, Canada) for germination and growth.

Inside the growth chamber, the following conditions were maintained for the day/night cycle: temperature of $31 / 27^{\circ} \mathrm{C}$, relative humidity at 55 to $65 \%$, and a light intensity of 260 to $350 \mu \mathrm{mol} \mathrm{m}^{-2} \mathrm{~s}^{-1} / 0 \mu \mathrm{mol} \mathrm{m}^{-2} \mathrm{~s}^{-1}$ at $12 / 12 \mathrm{~h}$. Following the germination of the seeds, seedlings were kept in plastic cups (60 ml volume) with $40 \mathrm{ml}$ distilled water and two seedlings were placed in each cup. Root system of plants was kept in water with nutrient solution over the whole growing period. Plant root system was exposed to liquid nutrient solution $\left(0.5 \mu \mathrm{lml}^{-1}\right.$ per plant), HYPONeX (Hyponex Japan Corp., Ltd., Japan), from first 1 week of seed germination, and watered along with the liquid fertilizer regularly over the whole duration of the experiments. For SIT measurement with 2,4-D, TIBA, and $\mathrm{GA}_{3}$, second latest leaves of fourteen to sixteen days old healthy seedlings were used as samples.

\section{Auxin and TIBA treatment protocol}

2,4-D (Wako Pure Chemical Industries, Ltd., Japan, molecular weight $220.04 \mathrm{~g} \mathrm{~mol}^{-1}$ ) was added to the distilled water to prepare three different concentrations 1,2 , and $4 \mu \mathrm{M}$ along with control or $0 \mu \mathrm{M}$. Two concentrations of 10 , and $20 \mu \mathrm{M}$ were prepared from TIBA (Tokyo chemical industry Co., Ltd., Tokyo, Japan, molecular weight $499.81 \mathrm{~g} \mathrm{~mol}^{-1}$ ). For each of the 2,4-D and TIBA concentrations, three leaf samples from three different plants were used in the measurement.

For 2,4-D exposure experiment, plants root system was kept in water for first $2 \mathrm{~h}$. Next, for the remaining $22 \mathrm{~h}$, the root systems were immersed in three different concentrations 1 , 2 , and $4 \mu \mathrm{M} 2,4-\mathrm{D}$. SIT observation was conducted from leaf for $24 \mathrm{~h}$ which includes two hours of control and $22 \mathrm{~h}$ under exposure of 2,4-D as shown in the protocol of Figure 1A. Under control or $0 \mu \mathrm{M}$, SIT observation was conducted for $24 \mathrm{~h}$ with root system exposed in water. In case of TIBA exposure same procedure was followed as that of 2,4-D exposure under 10 , and $20 \mu \mathrm{M}$ as shown in Figure 1A. Apart from NIF, leaf length was measured for 2,4-D, and TIBA, and the result is given as supplement.

\section{$\mathrm{GA}_{3}$ treatment protocols}

Gibberellic acid, $\mathrm{GA}_{3}$ (Wako Pure Chemical Industries, Ltd., Japan, molecular weight $346.4 \mathrm{~g} \mathrm{~mol}^{-1}$ ), was added to distilled water to prepare four different concentrations, $0,10,40$, and $100 \mu \mathrm{M}$. For every $\mathrm{GA}_{3}$ concentration, four leaf samples from four different plants were used in the measurement.

From the experimental protocol shown in Figure 1B, for the first $1 \mathrm{~h}$, plant root system was exposed in water. After this period, the root system was exposed to different concentrations of $\mathrm{GA}_{3}$ and measurements for the leaf were done for the next $5 \mathrm{~h}$. SIT observation was conducted for $6 \mathrm{~h}$ which includes one hours of control and $5 \mathrm{~h}$ under exposure of $\mathrm{GA}_{3}$.

In another experiment where $\mathrm{GA}_{3}$ was exposed over a long term, root systems of rice seedlings were continuously exposed to $0,10,40$, and $100 \mu \mathrm{M} \mathrm{GA}_{3}$ from 4 th to 22 nd day. From 10 th day of the plant age, SIT measurements were conducted for $2 \mathrm{~h}$ under $\mathrm{GA}_{3}$ exposure. These measurements were continued at 13th, 16th, and 19th day of plant age. For SIT observation, four samples were used under each concentration. This same set of samples was also used for leaf and root length measurements (Supplementary Figure S1), from 4th to 22nd day of the plant age with an interval of 2 days. SIT observation and length

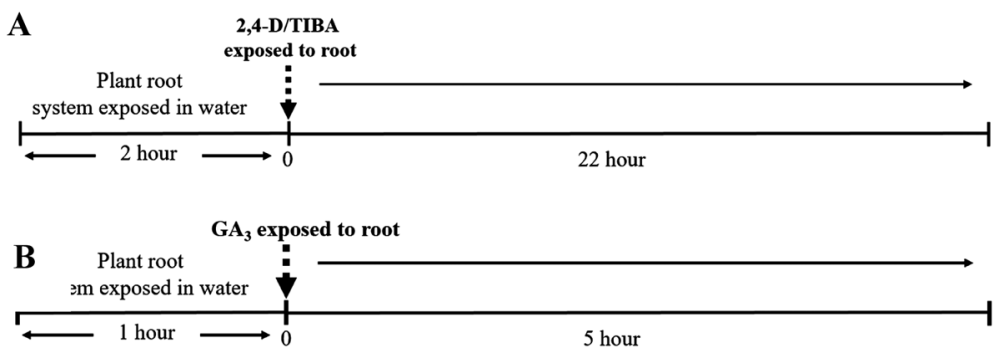

Figure 1. Experimental timing protocol for root system exposure to plant hormones with A. 2,4-D, and TIBA within $24 \mathrm{~h}$ and B. GA 3 within 6 h. 
A

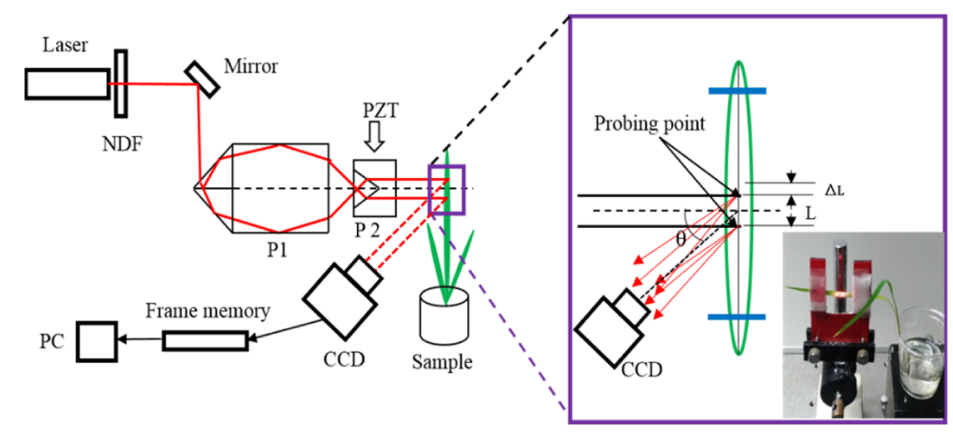

B

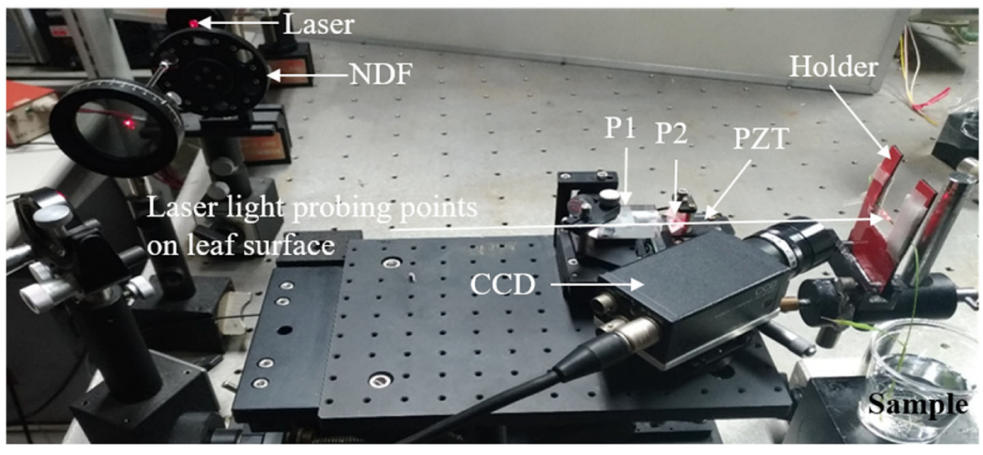

Figure 2. A schematic of the SIT system used in this study with an inset photograph of leaf attachment with holder (A), a photograph of the SIT system. Plant leaf is stably and gently placed in the holder (B). The illumination point of laser light was about $2.5-3 \mathrm{~cm}$ apart from leaf apex, and the distance between two probing laser beams was $3 \mathrm{~mm}$. NDF, neutral density filter; P1 and P2, prism; PZT, piezoelectric transducer; CCD, chargecoupled device.

measurements are given in the supplementary materials.

\section{Statistical interferometric technique and the experimental system}

A schematic of the experimental system of a statistical interferometer for measuring in-plane expansion of a leaf is shown in Figure 2A with an inset photograph showing leaf attached to a holder and a photograph of the SIT system in Figure 2B on an optical bench. As a light source, a He-Ne laser of wavelength $632.8 \mathrm{~nm}$ (GLG 5400, NEC Corporation, Japan) was used. At first, light was passed through a neutral density filter (F71N-2 Suruga Seiki, Japan) to adjust the level of the light intensity on the leaf surface. Next, using a specially designed prism, P1, the laser beam was divided into two beams. A rightangle prism, P2, mounted on a piezoelectric transducer (PZT) stage (E-620, Physik instrument, Germany) was employed to introduce path difference between the two illuminating beams. Plant leaf was mounted with a custom-made holder to measure the in-plane elongation or shrinkage of the surface of the leaf between the two probing points of the laser beam. It should be pointed out that base and the tip of the leaf were gently placed within the clamping gaps of the holder. To avoid damage to the leaf, cotton was used to cover the holder. The measurement position was around $2.2 \mathrm{~cm}$ from either side of the clamping positions. The leaf elongation was in the order of a few tens of micrometer within $24 \mathrm{~h}$ and this in comparison to the clamping positions is around 1,000 times smaller and so the clamp is expected to have no influence on the measurement.
When leaf surface is illuminated by laser light, a fully developed speckle pattern is generated (refer to Figure 3, De Silva et al. 2016) due to a random interference of the scattered light from the rough leaf surface. A charge-coupled device (CCD) (XC-75, Sony Corp., Japan, $768 \times 494$ pixel) was used to acquire the speckle interference pattern between the speckle fields generated from two probing beams. Generally, with leaf, a living biological tissue, two different kinds of speckle patterns arise from the scattered light. One is generated from the leaf surface and, the other from the laser light that penetrates deeper into the leaf. In SIT measurements, the speckles that are from the surface has to be used in measurements while suppressing those from within the leaf.

The speckles originating from within the leaf are due to the moving scatterers such as organelles within the leaf and are also known as biospeckles (Goodman 2007), and the intensity of such speckles vary with time. Such fluctuation in intensity of the biospeckles would result in degradation of SIT measurements, and thus the biospeckle has to be reduced. To reduce this biospeckle variation, the laser beam that is penetrating within the leaf has to be reduced. In order to reduce the penetration, illuminating area was covered with wheat flour. In real time measurement, contribution of laser biospeckle generation was confirmed to be less than $0.04 \%$ of the incident probing beams (Thilakarathne et al. 2014b).

\section{SIT principle and data acquisition}

SIT is an optical interferometer which can significantly measure 
A

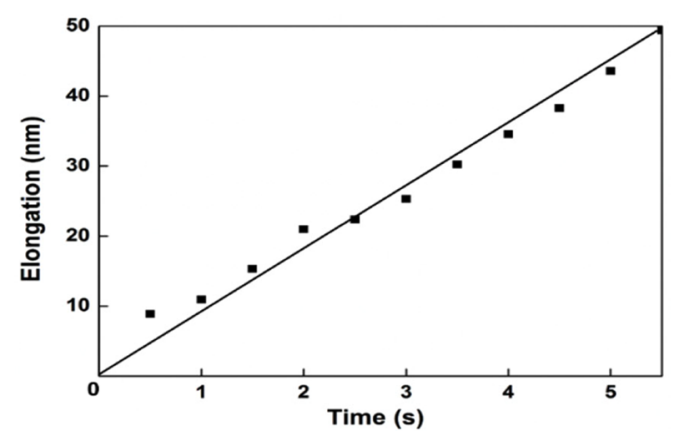

B

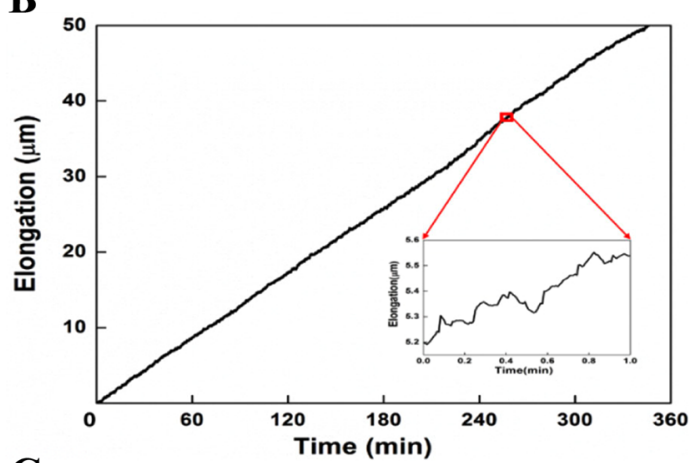

C

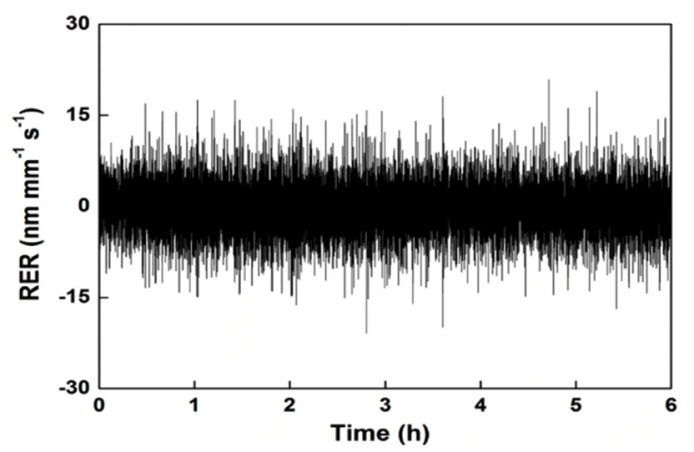

Figure 3. Rice plant leaf elongation at different time scales, (A) monotonous growth over $360 \mathrm{~min}$ with an inset of random fluctuations over $1 \mathrm{~min}$, (B) magnification over a $5.5 \mathrm{~s}$ of the inset with a linear regression fitted straight line to determine the slope that provides the RER, and (C) RER observed over the total duration of measurement of $6 \mathrm{~h}$ at different time scales by SIT under control.

short term growth dynamics of plant in nanometric scale in non-contact and a non-destructive way (Kadono and Toyooka 1991; Kadono et al. 2001). The principle of SIT is quite different in comparison to the conventional interferometers (Hariharan 1985). In conventional interferometers, phase change is obtained by analyzing an interference fringes generated by light reflected from an optically flat reference mirror and the one reflected from a specular surface under observation.

However, when a coherent light illuminates an optically rough surface, such as a leaf surface, a fully developed speckle field that shows a granular random pattern is generated instead of interference fringes. Therefore, the conventional interferometry cannot be applied any more. The probability density function (PDF) of phase of the fully developed speckle field has uniform probability density over the range from $-\pi$ to $\pi$. This statistical property of the speckle phase is stable and independent from the fine surface variations of the object surface. Therefore, instead of using the deterministic phase in the conventional interferometry, the statistical property of speckle field could be considered as a reference for object phase determination, and this forms the basis of SIT principle. Inplane displacement of biological or scattering surface object had been successfully measured in previous experimental studies (De Silva et al. 2017; Rathnayake et al. 2007; Thilakarathne et al. 2014a) and from such displacements in actual case, elongations of plant leaves were measured.

Two points on the surface of the leaf were illuminated by two parallel beams that were normal to the surface of the leaf plane. Two speckle fields were generated independently on the illuminating two points on leaf surface and random interference of speckle pattern originated. Power of individual illuminating beam on each spot of leaf surface was nearly $412 \mu \mathrm{W}$ and the size of an individual spot was kept $0.5 \mathrm{~mm}$. When this light beam illuminated the leaf surface penetration of light also occurred into the leaf. As a result of light penetration into leaf, dynamic speckle pattern or biospkeckles (Aizu and Asakura 1996) generated and to avoid light penetration into the leaf, wheat flour was used to cover the probing area.

To measure the amount of light transmitted through the wheat flour layer to address the phenomenon of light penetration investigation was conducted and found to be $2 \%$ of the incident light. For biospeckles generation involvement of light was much less than $0.04 \%$ of the incident illuminating beam as both the incident light and scattered light passed through the same flour layer. In case of the measurement of nanometric displacements the effect of wheat specially the displacement from the flour particles were found to be negligible (refer to Supplementary Figure S4, Thilakarathne et al. 2014a). In our experiment a $\mathrm{He}-\mathrm{Ne}$ laser, wavelength of $632.8 \mathrm{~nm}$ was used and found this wavelength was very close to the chlorophyll absorption band in spite of the penetrated light power being fairly small. Different experiments with the light source of wavelength 532 and $633 \mathrm{~nm}$ were performed to confirm the effect of the wavelength on plant growth properties (Thilakarathne et al. 2014a, b) and found the incident of illuminating beams did not affect the growth dynamics of the leaf. Experiments under environmental stress for heavy metal (De Silva et al. 2016) and micronutrient (De Silva et al. 2017) were also observed no visible injuries on leaf surface from illuminated light beams. In this study we have measured in-plane elongation on leaf surface between the two-probing illuminating beam for $24 \mathrm{~h}$ and no visible effect of laser illumination was observed on leaf surface over this time period. Here, we give a brief description of the method. The details regarding the measurement and the details of the actual data processing and analysis could be found in (Thilakarathne et al. 2014b).

Interference speckle patterns generated from the two illuminating points were acquired by a CCD camera for every $500 \mathrm{~ms}$ and stored in a frame memory. When $\Delta L$ is the in-plane 
elongation between the two illuminating points on the leaf plane, the phase difference between the two interfering speckle fields changes by $\Delta \varphi$ and can be expressed as,

$$
\Delta \varphi=\frac{2 \pi}{\lambda} \Delta L \sin \theta
$$

where $\lambda$ is the wavelength of laser light, and $\theta$ is the angle between the illumination and observation directions, and the actual values of $\lambda$ and $\theta$ were $633 \mathrm{~nm}$ and $17^{\circ}$, respectively. An algorithm described by Kobayshi and Kadono (2010) was used to determine the object phase $\Delta \varphi$. Leaf elongation, $\Delta L$, derived from Eq. (1) changes approximately linearly with time over a period $\tau$ of $5.5 \mathrm{~s}$, and from the slope, $\alpha\left(\mathrm{nm} \mathrm{s}^{-1}\right)$, of the regression line of elongation data for every $5.5 \mathrm{~s}$, relative elongation rate (RER) was defined by,

$$
R E R=\frac{\alpha}{L}
$$

where $L$ is the distance between two probing points and was kept being $3 \mathrm{~mm}$.

RER was calculated over the whole duration of measurement using Eq. (2), and special attention was also paid to the shortterm fluctuations of RER. In order to obtain RER and its fluctuations, following procedure was applied. At first, a linear regression was done over $5.5 \mathrm{~s}$ to obtain the slope, $\alpha$, or to obtain instantaneous RER, and the procedure was repeated for every $5.5 \mathrm{~s}$ across the whole measurement session to calculate the RER. Next, the calculated RERs were smoothened by passing through a moving average filter of a window size $165 \mathrm{~s}$ to obtain the smoothened data. Finally, the smoothened data was subtracted out from the instantaneous RER data calculated from linear regression over $5.5 \mathrm{~s}$. This was done to eliminate the long-term trend (De Silva et al. 2017; Thilakarathne et al. 2014b). The processed RER data was found to have fluctuations in the nanometric scale, and these fluctuations, named as nanometric intrinsic fluctuation (NIF), form the basis of our study. NIF was found to be an intrinsic characteristic determined by the type of the plant as well as the status of the plant such as young or old. Such intrinsic fluctuations in the nanometric scale were found to be completely absent in a dead leaf (refer to Figure 2, Thilakarathne et al. 2014b).

Standard deviations (SDs) of NIF were calculated to evaluate the dependence of NIF on the exogenous hormonal changes. Normalization was done to eliminate the variations between the sample differences during a single session. In the case of 2,4-D, and TIBA exposure experiments, first 2 hour of SIT measurement was done under control condition. After $2 \mathrm{~h}$, root system was exposed to 2,4-D, and TIBA, and elongation was measured for the next $22 \mathrm{~h}$ to calculate NIF. Control data for first $2 \mathrm{~h}$ was used for normalization of SD of the next $22 \mathrm{~h}$.

Under $\mathrm{GA}_{3}$ exposure experiment, first $1 \mathrm{~h}$ of SIT measurement was done under control or $0 \mu \mathrm{M} \mathrm{GA}_{3}$. After $1 \mathrm{~h}, \mathrm{GA}_{3}$ was exposed to root system of plant, and NIF was monitored for the next $5 \mathrm{~h}$ by SIT. Control data obtained over the first $1 \mathrm{~h}$ was used for normalization of SD of next five hours NIF.
The normalized SDs of NIF of total number of samples at each concentrations of 2,4-D, TIBA, and $\mathrm{GA}_{3}$ were averaged and referred to as the averaged normalized standard deviation (ANSD). One-tailed Students $t$-test was performed to analyze the statistical significance of the SD of NIF and ANSD obtained under different concentrations in comparison to those obtained from control or zero concentration of 2,4-D, TIBA, and $\mathrm{GA}_{3}$. Data analyses were done with Matlab (R2017b, MathWorks, Natick, USA), and graphs were prepared with Originlab (Origin Pro 8, Northampton, USA).

In case of continuous exposure experiments of $\mathrm{GA}_{3}$ over 19 days, four samples were chosen for SIT measurement, and ANSD (shown in Supplementary Figure S5) was calculated from the normalized SDs of NIF of four samples at each $\mathrm{GA}_{3}$ concentration.

\section{Results and discussion}

In our study, we have measured NIF by using SIT and also measured the macro parameters of leaf and root lengths under the exposure of plant hormones, 2,4-D, and $\mathrm{GA}_{3}$, and auxin transport inhibitor TIBA. Results of leaf and root length measurement are given as supplementary material.

\section{SIT measurements}

Figure 3 shows the elongation measurement of a rice leaf at different time scales by SIT. From the measured displacements or elongations, short-term relative elongation rates (RER) were calculated using Eq. (2) and were in the nanometric order per second. In order to calculate RER, at first, the in-plane displacement between the probing beams was measured every $500 \mathrm{~ms}$ over the whole experimental duration of $6 \mathrm{~h}$. A monotonous elongation in order of a few tens of micrometers over 360 min appeared as shown in Figure 3A with an inset showing the magnification of elongation over a period of minute. As could be seen, random fluctuations appear and further magnification to within a scale to seconds, the elongation was found to be almost linear (Figure 3B. From linear regression, the slope of the elongation was calculated to give the short-term relative elongation rate across the whole measurement period and is shown in Figure 3C. As can be seen, RER varies across the measurement period indicating the presence of continuous elongation and shrinkage. Further, the variation appeared to be random. These fluctuations were termed as nanometric intrinsic fluctuations (NIF), and our earlier studies (De Silva et al. 2016, 2017; Kabir et al. 2019; Thilakarathne et al. 2014a, b) revealed that these fluctuations, NIF, are sensitive to the environment of the plant.

\section{NIF results under 2,4-D}

Typical variations of the nanometric intrinsic fluctuations (NIF) are shown in Figure 4, when 
$\mathbf{A}$

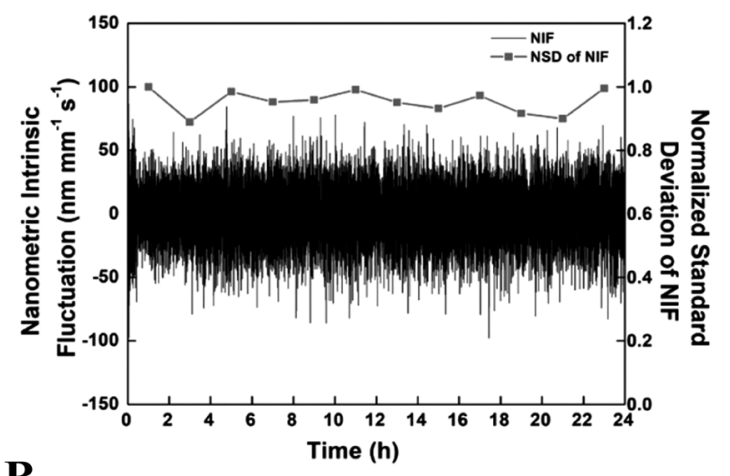

B

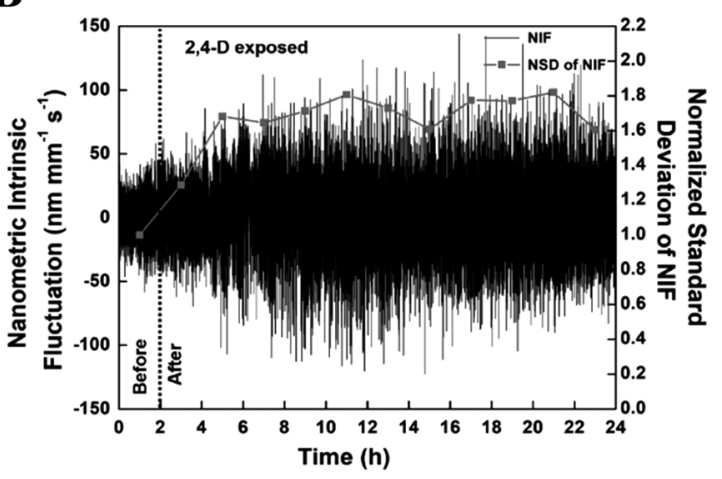

C

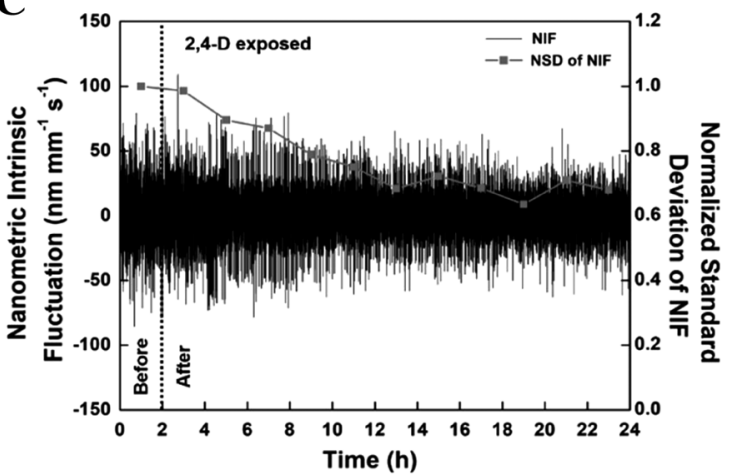

Figure 4. Variations of NIF (shown in black) and NSD of NIF (shown in gray) of rice leaf shown as a function of the measurement period under (A) control condition, (B) $1 \mu \mathrm{M}$, and (C) $4 \mu \mathrm{M} 2,4-\mathrm{D}$. The vertical dotted lines are drawn to show the start of the exposure of 2,4-D with "Before" and "After" indicating the exposure conditions. Note the fluctuations are in the order of nanometer indicating the sensitivity of SIT.

different concentrations of 2,4-D, 0 (control), 1, 2, and $4 \mu \mathrm{M}$ were applied to the rice root system. Normalized standard deviation (NSD) of the NIF was calculated by normalizing the SD of NIF under 2,4-D exposure with that at the initial $2 \mathrm{~h}$ under control condition. Under all concentrations, random fluctuations could be observed. However, depending on the concentration of 2,4-D, the fluctuations differ. No significant variation could be observed under the control condition seen in Figure 4A over the whole measurement period. At $1 \mu \mathrm{M} 2,4-\mathrm{D}$, NIF as seen in Figure $4 \mathrm{~B}$ increased within $1 \mathrm{~h}$ of exposure with the respective change of around from 23 to $40 \%$ at

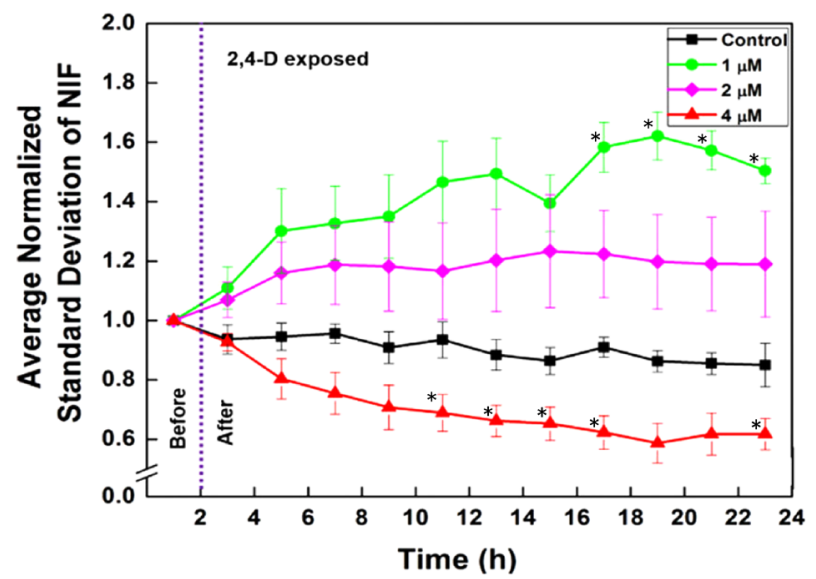

Figure 5. ANSD of NIF of rice leaf under exposure of four different concentrations of 2,4-D, 0 (black square), 1 (green circle), 2 (magenta diamond), and $4 \mu \mathrm{M}$ (red triangle) over $24 \mathrm{~h}$. The dotted line indicates the period of "Before" and "After" exposure of 2,4-D. Data were presented as average value of normalized SD from three sample as a function time. Error bars indicate standard errors (SE). One-tailed Students $t$-test $(p<0.05)$ was used to test the significant differences under all concentrations of 2,4-D compared to control. Asterisks $(*)$ indicate the statistically significant results under 1 , and $4 \mu \mathrm{M} 2,4$-D exposure with control.

$21 \mathrm{~h}$. NIF under $2 \mu \mathrm{M}$ 2,4-D was also increased over the whole exposure time but lower than that for $1 \mu \mathrm{M} 2,4-\mathrm{D}$ (shown in Supplementary Figure S6). Under $4 \mu \mathrm{M}$ 2,4D exposure, a gradual decrease in NIF (Figure 4C) was observed with a reduction rate around $30 \%$ at $21 \mathrm{~h}$.

ANSD of NIF under 0 (control), 1, 2, and $4 \mu \mathrm{M}$ concentrations of 2,4-D are shown in Figure 5. Under all the cases, it could be seen that the change in ANSD either increases or decreases happening within the first $1 \mathrm{~h}$ of the exposure to 2,4-D. Under the concentrations of 1 and $2 \mu \mathrm{M}$, there is increase in ANSD with respect to control. For 1, and $2 \mu \mathrm{M} 2,4-\mathrm{D}$, increments after $21 \mathrm{~h}$ of exposure from first $1 \mathrm{~h}$ were respectively 19 to $76 \%$, and 15 to $41 \%$. The increment for $2 \mu \mathrm{M}$ was lower than that for $1 \mu \mathrm{M}$ compared to control. In contrast, at $4 \mu \mathrm{M}$ of 2,4D, ANSD decreased with decrements to be respectively $15 \%$ and $27 \%$ following three and $21 \mathrm{~h}$ of exposure. Onetailed Students $t$-test was performed $(p<0.05)$ between control and each of the exposure 2,4-D concentrations of 1,2 , and $4 \mu \mathrm{M}$. Under 1 , and $4 \mu \mathrm{M}$ exposures, significant differences with respect to the control condition are indicated by the asterisk (*).

\section{NIF results under TIBA}

Results under TIBA, an auxin transport inhibitor, are shown in Figure 6 for 10 , and $20 \mu \mathrm{M}$. Under 10, and $20 \mu \mathrm{M}$ TIBA, from Figure $6 \mathrm{~A}$ and $6 \mathrm{~B}$, a gradual reduction in NIF was observed with a reduction rate of around $41 \%$ and $45 \%$, respectively, at $21 \mathrm{~h}$ of exposure.

The variation of ANSD of NIF under 0 (control), 10, and $20 \mu \mathrm{M}$ of TIBA is shown in Figure 7. Under both the concentrations of 10 , and $20 \mu \mathrm{M}$, there are decreases 
A

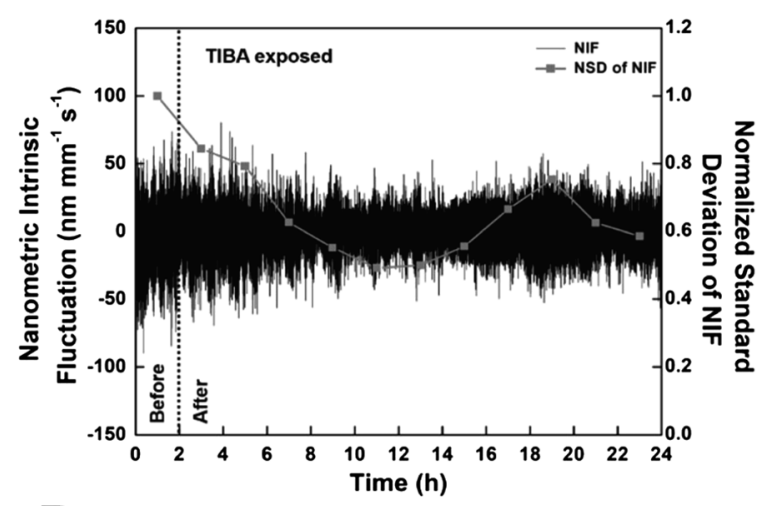

B

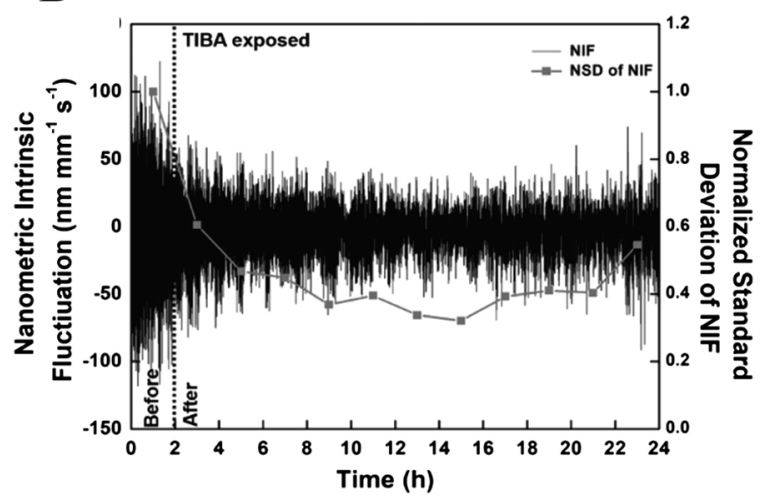

Figure 6. Variations of NIF (shown in black) and NSD of NIF (shown in gray) of rice leaf shown as a function of the measurement period under (A) $10 \mu \mathrm{M}$, and (B) $20 \mu \mathrm{M}$ TIBA. The vertical dotted lines are drawn to show the start of the exposure of TIBA with "Before" and "After" indicating the exposure conditions. Note the fluctuations are in the order of nanometer indicating the sensitivity of SIT.

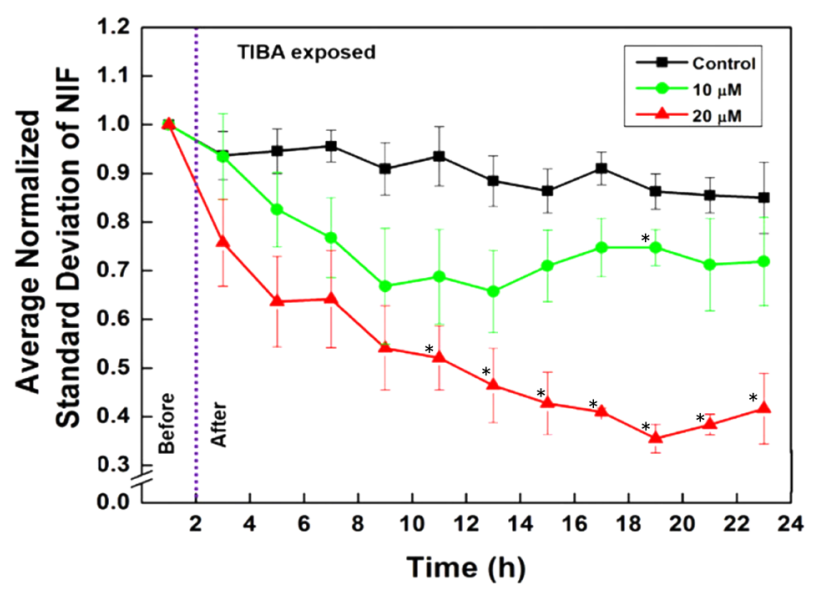

Figure 7. ANSD of NIF of rice leaf under exposure of three different concentrations of TIBA, 0 (black square), 10 (green circle), and $20 \mu \mathrm{M}$ (red triangle) over $24 \mathrm{~h}$. The dotted line indicates the period of "Before" and "After" exposure of TIBA. Data were presented as average value of normalized SD from three samples as a function time. Error bars indicate standard errors $(\mathrm{SE})$. One-tailed Students $t$-test $(p<0.05)$ was used to test the significant differences under all concentrations of TIBA compared to control. Asterisks (*) indicate the statistically significant results under 10 , and $20 \mu \mathrm{M}$ TIBA exposure with control. in ANSD with respect to the control. For $10 \mu \mathrm{M}$, ANSD decreased to $16 \%$ after $21 \mathrm{~h}$ of exposure from that at the first $1 \mathrm{~h}$. On the other hand, at a higher concentration of $20 \mu \mathrm{M}$ TIBA, ANSD decreased by $20 \%$ and $41 \%$ following one and $21 \mathrm{~h}$ respectively. One-tailed Students $t$-test was performed $(p<0.05)$ between control and each of the exposure TIBA concentrations of 10 , and $20 \mu \mathrm{M}$. Under 10 , and $20 \mu \mathrm{M}$ exposures, significant differences with respect to the control condition are indicated by the asterisk (*). Leaf length was also measured for 2,4-D and TIBA, and the results are given as Supplementary Figure S2 and S3 respectively.

\section{Results under short time exposure of $G A_{3}$}

In this experiment, for the first $1 \mathrm{~h}$, plant root system was exposed to water. After $1 \mathrm{~h}, \mathrm{GA}_{3}$ solution was added to the root system, and in-plane displacement was measured under $\mathrm{GA}_{3}$ exposure for the next $5 \mathrm{~h}$. NSD of the NIF was calculated by normalizing the SD of NIF under $\mathrm{GA}_{3}$ exposure with that at the initial one hour under control or $0 \mu \mathrm{M} \mathrm{GA}_{3}$. Under all the concentrations, random fluctuations could be observed as shown in Figure 8 .

From Figure $8 \mathrm{~A}$, it can be seen that no significant variation could be observed in NSD of NIF for the control condition over the whole measurement period. At $10 \mu \mathrm{M} \mathrm{GA}_{3}$, NIF of plants did not show significant change (data not shown) and was almost same as control. In contrast, as seen in Figure 8B, there was an increase in NIF under $40 \mu \mathrm{M} \mathrm{GA}_{3}$ within half an hour to four and half hours of exposure. Under $100 \mu \mathrm{M} \mathrm{GA}$ exposure, a gradual decrease in NIF (Figure 8C) was observed after four and half hours.

ANSD of NIF under 0 (control), 10,40 , and $100 \mu \mathrm{M}$ of $\mathrm{GA}_{3}$ are shown in Figure 9. Under low concentrations of 10 , and $40 \mu \mathrm{M}$, there was an increase in ANSD with respect to control. Under a high concentration of $100 \mu \mathrm{M}$, there was a decrease with decrement being less than that of control. The changes in ANSD was observed within the first half hour after the exposure to $\mathrm{GA}_{3}$ as compared to control. For $40 \mu \mathrm{M} \mathrm{GA}$, increment was monitored $3 \%$ at first half hour, to $8 \%$ after four and half hours of exposure. On the other hand, at a higher concentration of $100 \mu \mathrm{M} \mathrm{GA}_{3}$, decrements were $10 \%$ and $9 \%$ following 0.5 hour and 4.5 hours, respectively, and both decrements were significant. One-tailed Students $t$-test was performed $(p<0.05)$ between control and each of the exposure $\mathrm{GA}_{3}$ concentrations of 10,40 , and $100 \mu \mathrm{M}$. Under 40 , and $100 \mu \mathrm{M}$ exposures, significant differences with respect to the control are indicated by the asterisk $(*)$. Leaf and root length measurements were also conducted under $0,10,40$, and $100 \mu \mathrm{M}$ of $\mathrm{GA}_{3}$, and results are shown in Supplementary Figure S4. 
A
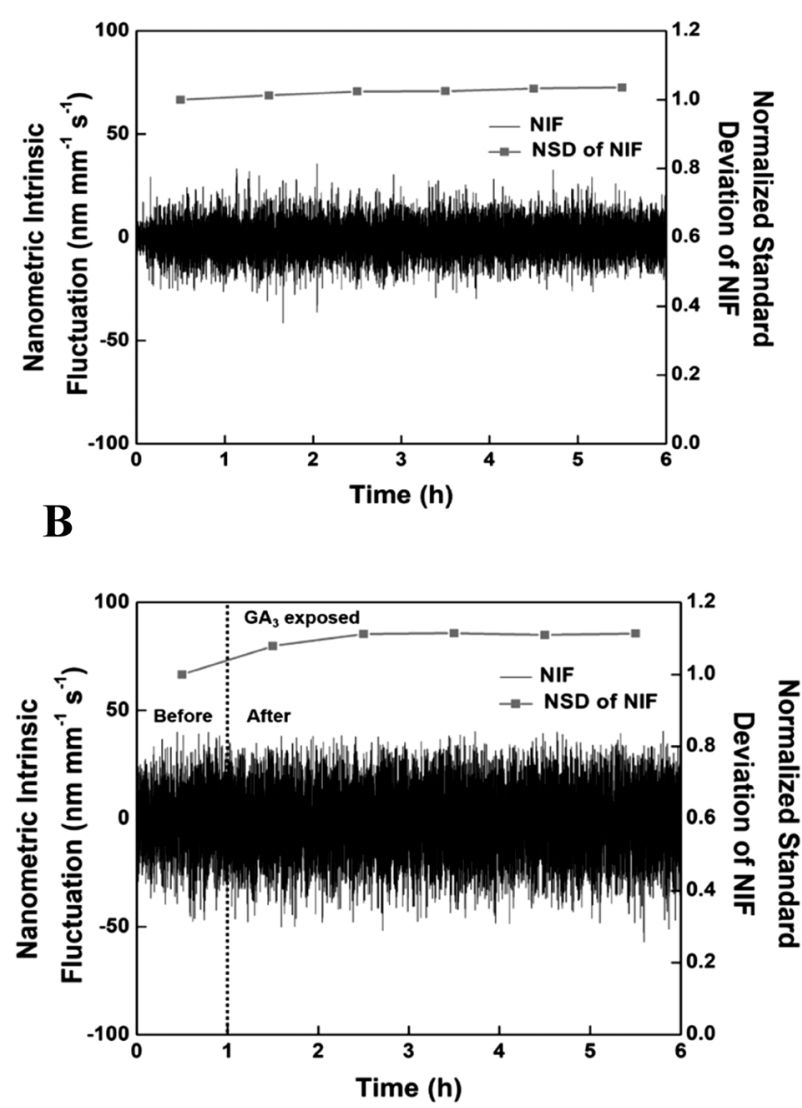

C

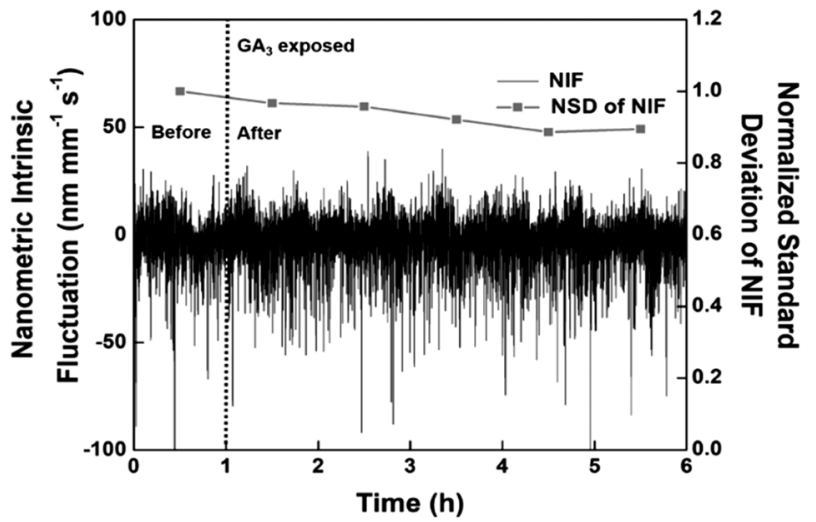

Figure 8. Variations of NIF (shown in black) and NSD of NIF (shown in gray) of rice leaf shown as a function of the measurement period under (A) control condition, (B) $40 \mu \mathrm{M}$, and (C) $100 \mu \mathrm{M} \mathrm{GA}_{3}$. The vertical dotted lines are drawn to show the start of the exposure of $\mathrm{GA}_{3}$ with "Before" and "After" indicating the exposure conditions. Note the fluctuations are in the order of nanometer indicating the sensitivity of SIT.

\section{NIF and plant growth hormones}

In our study, we have used an ultra-high accurate interferometer SIT to investigate the variation of RER under the presence of the plant growth hormones auxin, and $\mathrm{GA}_{3}$, along with auxin transport inhibitor TIBA. All the agents were applied to the root system of rice, and the elongations of the second latest leaf were measured

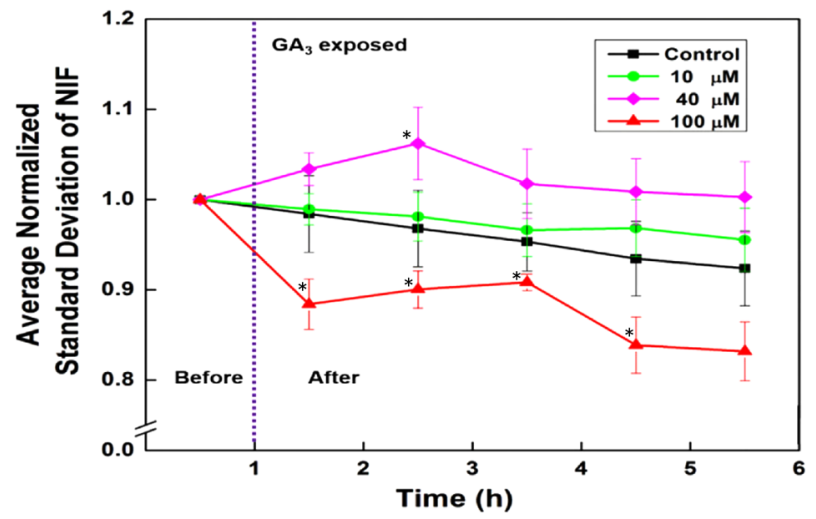

Figure 9. ANSD of NIF of rice leaf under exposure of four different concentrations of $\mathrm{GA}_{3,}, 0$ (black square), 10 (green circle), 40 (magenta diamond), and $100 \mu \mathrm{M}$ (red triangle) over $6 \mathrm{~h}$. The dotted line indicates the period of "Before" and "After" exposure of $\mathrm{GA}_{3}$. Data were presented as average value of normalized SD from four sample as a function time. Error bars indicate standard errors (SE). One-tailed Students $t$-test $(p<0.05)$ was used to test the significant differences under all concentrations of $\mathrm{GA}_{3}$ compared to control. Asterisks (*) indicate the statistically significant results under 40 , and $100 \mu \mathrm{M} \mathrm{GA}_{3}$ exposure with control.

under different concentrations. RER measurements after detrending the data revealed the presence of nanometric fluctuations that had been reported in our earlier studies too (De Silva et al. 2016, 2017; Kabir et al. 2019; Thilakarathne et al. 2014a, b). These fluctuations were found to vary depending on the hormones used.

In our previous studies, the reliability of NIF had been verified with a metal plate that was used as a rigid object instead of leaf and the level of fluctuations were almost a few tens to hundred times smaller than those of plant growth in the order of $0.1 \mathrm{~nm} \mathrm{~mm}^{-1} \mathrm{~s}^{-1}$ (Thilakarathne et al. 2014b). The fluctuations were also found to vary having different amplitudes depending on plants (Thilakarathne et al. 2014b). The measured random fluctuations were much larger than the noise level of the system, and thus the observed fluctuations arising out of spatial expansions happening at very fine scales across the leaf. The results showed the possibility that the nanometric RER fluctuations might be an intrinsic property of the plant and were not due to any external factors thus reflecting the microscopic processes. These results suggested that the fluctuations were clearly associated with the physiological state of the plant.

SIT was measuring the leaf elongation and the accompanying nanometric fluctuations during the process of elongation (De Silva et al. 2016; Kabir et al. 2019). The elongation can happen through cell expansion and cell division of the plant cell wall and this process is anisotropic with elongations occurring in three dimensions. However, SIT system can measure only in one directional in-plane with the plane of the leaf. The extension of the cell wall is controlled by a myriad of factors that makes the cell wall to expand and at the 
same time maintaining its integrity (Bashline et al. 2014). Different plant hormones are known to be involved in the control of the wall expansion (Nafisi et al. 2015).

Plant growth hormones are known to affect the orientation behavior of the microfibrils that make up the cell wall (Cosgrove 2014). Plant hormones both of auxin, and $\mathrm{GA}_{3}$, exposures induce radial cell expansion of microfibrils from a longitudinal to transverse orientation resulting from the inducement of the microtubules (Tanimoto 2005). Hormone induced microtubule reorientation and thus of cellulose fibril involves complex feedback mechanisms (Sampathkumar et al. 2014). It is possible that such fine controls involved in maintaining cell wall integrity during cell expansion could be possible in the origin of NIF. The results showed that NIF is high for certain concentrations. At concentrations of 1 , and $2 \mu \mathrm{M}$ of $2,4-\mathrm{D}$ and 10 , and $40 \mu \mathrm{M}$ of $\mathrm{GA}_{3}$, NIF increased in comparison to that of the control indicating the possibility of dominance of the cell expansion rather than from turgor pressure related. At high concentrations of $4 \mu \mathrm{M}$ of $2,4-\mathrm{D}$, and $100 \mu \mathrm{M} \mathrm{GA}_{3}$, ANSD reduced with reduction even lower than the control. This could be possibly due to factors that play to maintain the cell wall integrity which is necessary in accommodating neighboring cells resulting in large anisotropic stress over cell wall expansion (Hamant and Traas 2010). Further, by auxin transport inhibitor TIBA again introduced reduction of NIF with the reduction in ANSD getting larger with increase in the concentration of TIBA.

To conclude, in this study SIT has been used for monitoring the effect of plant hormone on rice seedlings and was proven to be capable in successfully monitoring the nanometric elongation under exogenous hormone exposure. From experimental studies with SIT, plant hormones as well as hormone transport inhibitors has immediate influence on nanometric fluctuations under different concentrations even within $1 \mathrm{~h}$ to several hours and thus SIT is sensitive enough to monitor nanometric growth behavior of plant. In this study it was found that, the NIF is sensitive to the elongation and shrinkage behavior of the cell wall. Our results strongly suggest that SIT can be used for speedily monitoring the effect of exogenous application of endogenic plant hormones auxin, and $\mathrm{GA}_{3}$, and auxin transport inhibitor TIBA on rice seedlings through the nanometric elongation fluctuations.

\section{Acknowledgements}

The authors are thankful to Dr. Yiheng Lim (Assistant Professor, Saitama University), Dr. Li Tao (Doctoral Student, Saitama University) for technical support.

\section{References}

Aizu Y, Asakura T (1996) Bio-speckles. In: Consortini A (ed)
Trends in Optics, 1st ed. Academic Press, San Diego, pp 27-49

Bashline L, Lei L, Li S, Gu Y (2014) Cell wall, cytoskeleton, and cell expansion in higher plants. Mol Plant 7: 586-600

Begum MC, Islam M, Sarkar MR, Azad MAS, Huda AKMN, Kabir AH (2016) Auxin signaling is closely associated with Znefficiency in Rice (Oryza sativa L.). J Plant Interact 11: 124-129

Botía JM, Ortuño A, Acosta M, Sabater F, Sánchez-Bravo J (1992) Influence of 2,3,5-triiodobenzoic acid on the transport and metabolism of IAA in Lupin hypocotyls. J Plant Growth Regul 11: $135-141$

Briers JD (1977) The measurement of plant elongation rates by means of holographic interferometry: Possibilities and limitations. J Exp Bot 28: 493-506

Brock TG, Kaufman PB (1988) Altered growth response to exogenous auxin and gibberellic acid by gravistimulation in pulvini of Avena sativa. Plant Physiol 87: 130-133

Cai T, Meng X, Liu X, Liu T, Wang H, Jia Z, Yang D, Ren X (2018) Exogenous hormonal application regulates the occurrence of Wheat tillers by changing endogenous hormones. Front Plant Sci 9: $1-17$

Clark DP, Pazdernik NJ (2016) Transgenic plants and plant biotechnology. In: Biotechnology. Elsevier, Amsterdam, pp 461-492

Cosgrove DJ (2014) Re-constructing our models of cellulose and primary cell wall assembly. Curr Opin Plant Biol 22: 122-131

Daie J (1987) Interaction of cell turgor and hormones on sucrose uptake in isolated phloem of Celery. Plant Physiol 84: 1033-1037

Davies PJ (2010) The Plant Hormones: Their Nature, Occurrence, and Functions. Springer Netherlands, Dordrecht

De Silva KTKM, Rajagopalan UM, Kadono H (2016) Monitoring of growth dynamics of plants under the influence of cadmium using a highly sensitive interferometry technique. Opt Eng 55: 121718

De Silva KTKM, Rajagopalan UM, Kadono H (2017) Highly sensitive optical interferometric technique reveals stressdependent instantaneous nanometric growth fluctuations of Chinese chive leaf under heavy metal stress. Ecotoxicol Environ Saf 137: 86-93

Dianty JC (1975) Topics in Applied Physics. Laser Speckle and Related Phenomena. Springer Berlin, Heidelberg

Durnam DJ, Jones RL (1982) The effects of colchicine and gibberellic acid on growth and microtubules in excised Lettuce hypocotyls. Planta 154: 204-211

Fox MD, Puffer LG (2008) Holographic interferometric measurement of motions in mature plants. Plant Physiol 60: $30-33$

Furukawa K, Yang YY, Honda I, Yanagisawa T, Sakurai A, Takahashi N, Kamiya Y (1997) Effects of ethylene and gibberellins on the elongation of rice seedlings (Oryza sativa L.). Biosci Biotechnol Biochem 61: 864-869

Goodman J (2007) Speckle Phenomena in Optics: Theory and Applications. Roberts \& Company Pub., USA

Hamant O, Traas J (2010) The mechanics behind plant development. New Phytol 185: 369-385

Hariharan P (1985) Optical Interferometry, 2nd ed. Academic Press, Sydney

Jiang Z, Stadue W (1989) An interferometric method for plant growth measurements. J Exp Bot 40: 1169-1173

Kabir M, Rajagopalan UM, Lim Y, Kadono H (2019, July 14-16) Visualizing the influence of growth hormones in plant by statistical interferometric and optical coherence tomography techniques. [Conference proceedings T1E-XI5] Optics and 
Photonics Congress, OSK- OSA-OSJ Joint Symposia, Busan, Korea. http://jointsymposia2019.osk.or.kr/?page_id=35

Kadono H, Toyooka S (1991) Statistical interferometry based on the statistics of speckle phase. Opt Lett 16: 883-885

Kadono H, Toyooka S, Iwasaki Y (2001) Speckle-shearing interferometry using a liquid-crystal cell as a phase modulator. J Opt Soc Am A Opt Image Sci Vis 8: 2001-2008

Karabaghli-Degron C, Sotta B, Bonnet M, Gay G, Le Tacon F (1998) The auxin transport inhibitor 2,3,5-triiodobenzoic acid (TIBA) inhibits the stimulation of in vitro lateral root formation and the colonization of the tap-root cortex of Norway spruce (Picea abies) seedlings by the ectomycorrhizal fungus Laccaria bicolor. New Phytol 140: 723-733

Kawamura H, Kamisaka S, Masuda Y (1976) Regulation of Lettuce hypocotyl elongation by gibberellic acid. Correlation between cell elongation, stress-relaxation properties of the cell wall and wall polysaccharide content. Plant Cell Physiol 17: 23-34

Keyes G, Sorrells ME, Setter TL (1990) Gibberellic acid regulates cell wall extensibility in Wheat (Triticum aestivum L.). Plant Physiol 92: 242-245

Kirpichnikova A, Chen T, Teplyakova S, Shishova M (2018) Proton pump and plant cell elongation. Biol Commun 63: 32-42

Kobayashi A, Hori K, Yamamoto T, Yano M (2018) Koshihikari: A premium short-grain rice cultivar-Its expansion and breeding in Japan. Rice (N Y) 11: 15

Kobayashi K, Kadono H (2010) Expansion of the dynamic range of statistical interferometry and its application to extremely short to long-term plant growth monitoring. Appl Opt 49: 6333-6339

Kučerová D, Kollárová K, Vatehová Z, Lišková D (2016) Interaction of galactoglucomannan oligosaccharides with auxin involves changes in flavonoid accumulation. Plant Physiol Biochem 98: 155-161

Majda M, Robert S (2018) The role of auxin in cell wall expansion. Int J Mol Sci 19: 951

Matsukura C, Itoh S, Nemoto K, Tanimoto E, Yamaguchi J (1998) Promotion of leaf sheath growth by gibberellic acid in a dwarf mutant of Rice. Planta 205: 145-152

Miceli A, Moncada A, Sabatino L, Vetrano F (2019) Effect of gibberellic acid on growth, yield, and quality of leaf Lettuce and Rocket grown in a floating system. Agronomy (Basel) 9: 382

Nafisi M, Fimognari L, Sakuragi Y (2015) Interplays between the cell wall and phytohormones in interaction between plants and necrotrophic pathogens. Phytochemistry 112: 63-71

Pohanish RP (2015) In: Pohanish RPBT-SH of P and AC (ed) Sittig's Handbook of Pesticides and Agricultural Chemicals, 2nd ed. Elsevier, Oxford, pp 196-331

Rathnayake AP, Kadono H, Toyooka S, Miwa M (2007) Statistical interferometric investigation of nano-scale root growth: Effects of short-term ozone exposure on ectomycorrhizal pine (Pinus densiflora) seedlings. J For Res 12: 393-402

Rathnayake AP, Kadono H, Toyooka S, Miwa M (2008) A novel optical interference technique to measure minute root elongations of Japanese red pine (Pinus densiflora Seibold \& Zucc.) seedlings infected with ectomycorrhizal fungi. Environ Exp Bot 64: 314-321

Sampathkumar A, Yan A, Krupinski P, Meyerowitz EM (2014) Physical forces regulate plant development and morphogenesis. Curr Biol 24: R475-R483

Shi F, Takasaki H, Komatsu S (2008) Quantitative analysis of auxinregulated proteins from basal part of leaf sheaths in Rice by two- dimensional difference gel electrophoresis. Phytochemistry 69: 637-646

Shibaoka H (1993) Regulation by gibberellins of the orientation of cortical microtubules in plant cells. Funct Plant Biol 20: 461

Shinkle JR, Briggs WR (1984) Auxin concentration/growth relationship for Avena coleoptile sections from seedlings grown in complete darkness. Plant Physiol 74: 335-339

Sosnowski J, Malinowska E, Jankowski K, Król J, Redzik P (2019) An estimation of the effects of synthetic auxin and cytokinin and the time of their application on some morphological and physiological characteristics of Medicago x varia T. Martyn. Saudi J Biol Sci 26: 66-73

Taiz L, Zeiger E (2003) Gibberellins: Regulators of plant height. In: Lazar T (ed) Plant Physiology, 3rd ed. Sinauer Associates Inc., Sunderland, Massachusetts, pp 461-492

Takahashi K, Hayashi Ki, Kinoshita T (2012) Auxin activates the plasma membrane $\mathrm{H}+$-ATPase by phosphorylation during hypocotyl elongation in Arabidopsis. Plant Physiol 159: 632-641

Takeno K, Pharis RP (1982) Brassinosteroid-induced bending of the leaf lamina of dwarf Rice seedlings: An auxin-mediated phenomenon. Plant Cell Physiol 23: 1275-1281

Tanimoto E (2005) Regulation of root growth by plant hormonesroles for auxin and gibberellin. CRC Crit Rev Plant Sci 24: 249-265

Tanimoto E (2012) Tall or short? Slender or thick? A plant strategy for regulating elongation growth of roots by low concentrations of gibberellin. Ann Bot 110: 373-381

Tanimoto E, Huber DJ (1997) Effect of GA3 on the molecular mass of polyuronides in the cell walls of Alaska pea roots. Plant Cell Physiol 38: 25-35

Taylor A, Cosgrove DJ (1989) Gibberellic acid stimulation of Cucumber hypocotyl elongation. Plant Physiol 90: 1335-1340

Thilakarathne BL, Rajagopalan U, Kadono H, Yonekura T (2014a) An optical interferometric technique for assessing ozone induced damage and recovery under cumulative exposures for a Japanese rice cultivar. Springerplus 3: 89

Thilakarathne BLS, Rajagopalan UM, Kadono H, Yonekura T (2014b) High speed and high precision optical interferometric technique to investigate instantaneous growth related changes of plant leaves. Plant Biotechnol 31: 195-205

Tiwari B, Kharwar S, Tiwari DN (2019) Pesticides and rice agriculture. In: Mishra AK, Tiwari DN, Rai AN (eds) Cyanobacteria. Elsevier, London, pp 303-325

Valery N, Jane K, Theodore A, Peter YS, Bancy W, Isidore M, Jean N, Sindi K, Damien S, Peter N, et al. (2014) In vitro effects of gibberellic acid and sucrose concentration on micropropagation of two elite sweet potato cultivars in Rwanda. Int J Biotechnol Mol Biol Res 5: 1-6

Yamaguchi S (2008) Gibberellin metabolism and its regulation. Annu Rev Plant Biol 59: 225-251

Zhang Y, Yin H, Zhao X, Wang W, Du Y, He A, Sun K (2014) The promoting effects of alginate oligosaccharides on root development in Oryza sativa L. mediated by auxin signaling. Carbohydr Polym 113: 446-454

Zhao SQ, Xiang JJ, Xue HW (2013) Studies on the Rice leaf inclination1 (LC1), an IAA-amido synthetase, reveal the effects of auxin in leaf inclination control. Mol Plant 6: 174-187

Zhao Y (2010) Auxin biosynthesis and its role in plant development. Annu Rev Plant Biol 61: 49-64 